\title{
Identification and discrimination of handshape in American Sign Language
}

\author{
JAMES STUNGIS \\ Northeastern University, Boston, Massachusetts 02115
}

\begin{abstract}
Lane, Boyes-Braem, and Bellugi (1976) suggested that American Sign Language (ASL) was perceived according to distinctive features, as is the case with speech. They advanced a binary model of distinctive features for one component of ASL signs, handshape. To test the validity of this model, three native users of ASL and three English speakers who knew no ASL participated in two experiments. In the first, subjects identified ASL handshapes obscured by visual noise, and confusion frequencies yielded similarity scores for all possible pairs of handshapes. Pairs that shared more features according to the model produced higher similarity scores for both groups of subjects. In the second experiment, subjects discriminated a subset of all possible handshape pairs in a speeded "same-different" task; discrimination accuracy and reaction times yielded a $\mathrm{d}^{\prime}$ and a $\mathrm{d}_{\mathrm{s}}$ value, respectively, for each pair. Pairs that shared more features according to a slightly revised version of the model produced lower discrimination indices for both groups. While the binary model was supported, a model in which handshape features varied continuously in two dimensions was more consistent with all sets of data. Both models describe hearing and deaf performance equally well, suggesting that linguistic experience does little to alter perception of the visual features germane to handshape identification and discrimination.
\end{abstract}

American Sign Language (ASL) is the manual, visual language used by the deaf community in the United States. It is not finger-spelling, pantomime, or English on the hands. ASL is a systematic natural form of communication among a stable community of users. The language is transmitted by deaf parents to their deaf (and some hearing) children and has the degree of regularity and structure required of a fully developed language (Battison, 1974; Bellugi, Klima, \& Siple, 1975; Frishberg, 1975; Klima \& Bellugi, 1979; Woodward, 1974; Grosjean, Teuber, \& Lane, Note 1; Stokoe, Note 2).

Spoken and sign languages show certain resemblances in forming lexical items. A spoken language forms words from a closed set of formational elements called phonemes. English constructs words from a set of 38 phonemes partitioned into two classes of formational parameters: consonants ( 22 phonemes), characterized by a constriction formed at some point in the vocal tract, and vowels (16 phonemes), characterized by a pattern of openness of the vocal tract (Trager \& Smith, Note 3).

This research was supported by grants from the National Science Foundation (768 2530) and the Department of Health, Education and Welfare (NS14923), and by a predoctoral fellowship from the National Institute of Mental Health (1 F31 MH 07492-01). The author would like to thank Francois Grosjean, Harlan Lane, and Joanne Miller for their helpful comments and criticisms. Requests for reprints should be sent to Jim Stungis, Department of Psychology, Northeastern University, 360 Huntington Avenue, Boston, Massachusetts 02115.
Sign language forms signs from a closed set of formational elements called primes. ASL constructs signs from a set of 62 primes partitioned into four classes of formational parameters: handshape (20 primes), characterized by the pattern of the fingers relative to the palm of the hand; location (14 primes), the position of the hand relative to specific parts of the body; orientation (4 primes), the direction of the palm of the hand relative to the body; and movement (24 primes), the motion of the hand relative to the body and to the other hand (for discussion, see Battison, 1978).

Just as phonemes function to contrast minimally different words, primes function to contrast minimally different signs. Change the initial consonant of the English word "pin" to $/ \mathrm{b} /$ and then to $/ \mathrm{d} /$, keep all other formational elements constant, and two successive changes in the meaning of the word result. Changing the value of a single prime of a sign in ASL produces an analogous result. For example, in the sign transcribed by the English word CUTE, the handshape is closed with the index and middle fingers fully extended side by side ( $\mathrm{H}$ handshape; see Figure 1). The location is chin: extended fingertips contact the chin just below the center of the bottom lip. The palm orientation is toward the signer's body. The movement is linear, downward, twice repeated; during each downward excursion, extended fingertips maintain contact with the chin as they move across it. Change the handshape of the sign CUTE from $\mathrm{H}$ to $G$, keep all other formational elements the same, and the meaning of the sign changes to RED. Again 
change the handshape of the sign RED from $G$ to $B$, keep all other formational elements the same, and the meaning of the sign changes to SWEET.

Just as phonemes have been shown to be functional in the acoustic decoding of words (Conrad, 1962, 1964; Norman, 1970, 1976; Sperling \& Speelman, 1970), primes have been shown to be functional in the visual decoding of signs (Bellugi \& Siple, 1974; Bellugi, Klima, \& Siple, 1975; Klima \& Bellugi, 1979; Grosjean et al., Note 1). However, numerous studies of spoken language have demonstrated that phonemes are not the smallest units of linguistic processing (see Fromkin, 1971; Liberman, Cooper, Shankweiler, \& Studdert-Kennedy, 1967; Mackay, 1970; Nooteboom, Note 4). Nonrandom patterns of phonemic substitutions have occurred in a variety of experimental paradigms (Eimas \& Corbit, 1973; Miller \& Nicely, 1955; Wickelgren, 1965, 1966). The regularity of these patterns has supported the hypothesis that phonemes themselves are perceived according to distinctive features.

Lane, Boyes-Braem, and Bellugi (1976) found evidence that primes are perceived according to distinctive features. ${ }^{1}$ Using a procedure similar to that of Miller and Nicely (1955), Lane et al. varied each prime of the handshape parameter across three levels of the other three parameters and had signers identify the handshapes of these signs in noise. The ShepardKruskal nonmetric multidimensional scaling technique (Kruskal, 1964a 1964b; Shepard, 1962, 1972) and a method of cluster analysis devised by D'Andrade (1978) revealed that handshape substitutions were not random. The similarity structure which these analyses highlighted led the investigators to advance a model of 11 distinctive features functional in the visual decoding of handshape.

Lane et al. noted that their model predicted handshape confusion in visual noise better than Chomsky and Halle's (1968) consonant feature model predicted the consonant confusions in auditory noise from Miller and Nicely (1955). Lane et al. demonstrated the descriptive adequacy of their handshape feature model with respect to their own data. Discriminability indices derived from the model for all possible pairs of handshapes correlated well with the confusion frequencies of the pairs. The investigators also demonstrated the predictive power of their model with respect to independent sets of handshape substitution data. Handshapes involved in alternative pronunciations in the Dictionary of American Sign Language (Stokoe, Casterline, \& Croneberg, 1965), in memory errors (Bellugi et al., 1975; Bellugi \& Siple, 1974), and in "slips of the hand" (Newkirk, Pederson, \& Bellugi, Note 5) all shared significantly more features according to the model than would be expected by chance.

These demonstrations are not, however, con- vincing tests of the model's descriptive adequacy or its predictive power. With regard to descriptive adequacy, one would expect some ad hoc feature model to describe a set of empirical confusion data and apparently Lane et al. have found a good model for their data. But how general is the model? It may not, for two reasons, adequately describe the handshape confusion data of native signers that were not instrumental in establishing the model. First, the model is based on relatively few observations per handshape. Wickelgren $(1965$, p. 397) observed that estimating a large matrix of confusion frequencies requires a very large number of trials before the random error is so low that one can believe the results of every individual paired comparison. He advanced a consonant feature model, based on nearly 3,000 observations per consonant, that was more accurate than Miller and Nicely's (1955) model in describing the consonant confusions of both their studies. Yet Miller and Nicely's distinctive feature model did adequately describe their own data, and was based on a fair number of observations per consonant, 250 in each test condition. A distinctive feature model based on only 54 observations per segment, as is the Lane et al. model, may be a biased account of handshape perception with little generality.

Second, the model may not adequately describe the handshape confusions of native signers because it is not based on the observations of native signers. Both of Lane et al.'s subjects learned ASL as a second language. One subject was hard of hearing, the daughter of hearing parents, and did not learn ASL until age 11. The other subject was hearing until age $51 / 2$, the son of hearing parents, and did not learn ASL until age 6. Abramson and Lisker (1970), Goto (1971), Miyawaki, Strange, Verbrugge, Liberman, Jenkins, and Fujimura (1975), and Williams (1974) have all shown that, in speech, linguistic experience can alter the perception of an acoustic cue signifying contrasting linguistic segments. As a result, linguistically naive and linguistically sophisticated subjects exhibit different patterns of segment identification. Linguistic experience may have altered perception of the visual cues signifying linguistically contrastive handshapes in ASL. In this case, the Lane et al. model may adequately describe the handshape confusions of naive subjects and nonnative signers but inadequately describe the confusions of native signers.

The first experiment is a test of the generality of the model and an assessment of the effect of linguistic experience on handshape identification. It is a replication of the Lane et al. study in that it employs the same paradigm to collect handshape confusions. It is an extension of their study in that it collects nearly eight times as many observations per handshape and compares two groups of subjects not observed by Lane et al., nonsigners and native signers. If the 
distinctive feature model accurately describes the process of handshape identification in native signers, it should predict their pattern of handshape confusions. If, in addition, linguistic experience does nothing to alter perception of the visual cues signifying linguistically contrastive handshapes in ASL, the model should also predict nonsigners' handshape confusions.

With regard to the predictive power of the Lane et al. model, the authors provide only general demonstrations of the accuracy of the model when very specific demonstrations are possible. On the average, handshapes involved in alternative sign pronunciations and in memory and production errors share more features than would be expected by chance. Specifically, however, if we chose one handshape as a standard and several others as comparisons, would we observe a lower discrimination index the more features standard-comparison pairs shared when signers were induced into error and required to judge the similarity of the pairs? The model easily lends itself to this kind of a test. Yet it has never been performed by Lane et al. or others.

The second experiment is a specific test of the predictive power of the Lane et al. model. It employs a new paradigm, time compression, to induce subjects into error and thereby reveal the rank-order discriminability of several comparison handshapes with respect to selected handshape standards. If the model is an accurate discriptor of discrimination as well as identification, native signers should display lower discrimination indices as handshape pairs share more features according to the model. If linguistic experience is not necessary to consistently use the same set of visual features across tasks commonly requiring handshape differentiation, nonsigners should display the same rank order of discrimination indices as do signers.

\section{EXPERIMENT 1}

\section{Method}

Subjects. Three native signers and three nonsigners participated for pay in the experiment. The native signers $(49,41$, and 23 years of age; all female) were congenitally deaf and had learned ASL as a first language from their deaf parents. The nonsigners $(22,20$, and 21 ; two males and one female, respectively) had normal hearing and no experience with ASL. One of the signers had normal corrected vision; all other subjects had normal uncorrected vision.

Stimuli and Apparatus. The same videotapes employed by Lane et al. were used in this experiment. So that stimulus items would display the form of signs as they occur in natural communication, each of the 20 primes of the handshape parameter (see Figure 1) was varied over three primes of each of the other three parameters. This resulted in a random sequence of 540 signs that are formationally acceptable but have no meaning in ASL (20 handshapes $x$ three locations $x$ three palm orientations $x$ three movements). Letters are used here to designate the ASL handshapes according to the code used in Stokoe et al. (1965). The location primes were (1) mouth, (2) upper left arm, and (3) neutral space, about $15 \mathrm{~cm}$ in front of the signer's right shoulder. The orientation primes
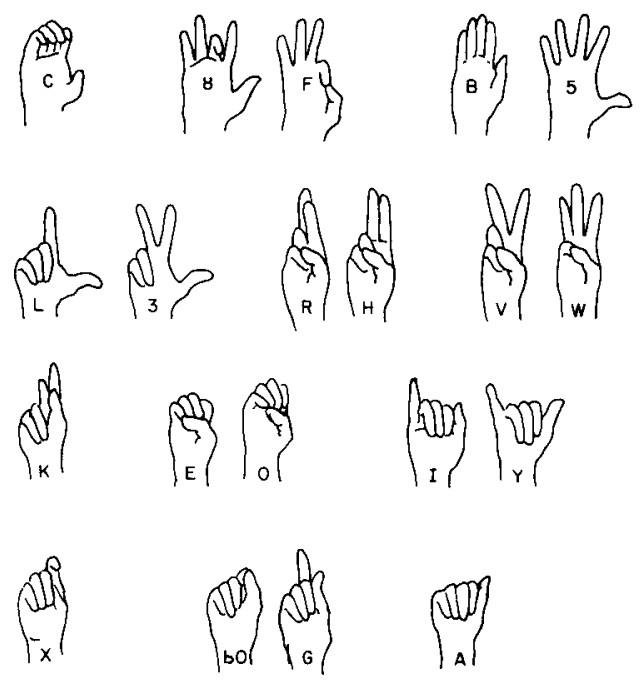

Figure 1. Inventory of handshape primes in signs used in experiments on identification and discrimination.

were (1) palm facing away from the signer, (2) palm toward the signer, and (3) palm down. The movement primes were (1) two medial twists of the wrist, (2) a clockwise circular movement of the arm beginning near the signer and moving away, and (3) a repeated left-to-right sweeping movement of the arm. These signs were performed by a native signer and recorded on a video tape. Each sign was $1 \mathrm{sec}$ in duration followed by a 4-sec blank. After every fifth sign, an additional 4-sec blank was inserted to help subjects keep their written responses in correspondence with the stimuli.

Noise in the form of video "snow" appeared $.5 \mathrm{sec}$ before each sign and continued throughout presentation of the sign. The distorted test tape was prepared by mixing (special effects generator, Sony SEG 1) noise from an idling video recorder (Sony AV-3600 while the undistorted signs played back from a second recorder (Sony AV-3650). Peak-to-peak signal and noise levels were read from an oscilloscope and mixed in nearly equal proportions $(.96$ or $-.35 \mathrm{~dB} \mathrm{~S} / \mathrm{N})$. Seated $2 \mathrm{ft}$ from the stimulus display placed at eye level, subjects viewed the test tape on an 8-in. video monitor (Sony CVM 950) whose brightness and contrast controls were set at maximum. Fluorescent overhead room lighting produced $41-\mathrm{fc}$ illumination.

Procedure. Each subject participated in five identification sessions, 540 trials per session, for a total of 135 observations per handshape per subject $(5 \cdot 540 / 20=135)$. A subject was given a 20 min break after viewing half the test tape and 10 -min breaks at the one-fourth and three-fourths marks. The test tape was begun at a different randomly determined starting point in each new session.

The subjects were told that the palm orientation, location, and movement of the sign would vary, but that their task was to identify the handshape by writing a symbol. Prior to the experiment, the subjects were given pictures of the 20 handshapes and the corresponding written symbols to be used in identification. Next, subjects viewed a clear, undistorted version of the test tape repeatedly until they demonstrated better than $95 \%$ accurate use of the written symbol. The subjects met this criterion in two presentations, at most, of the $\mathbf{5 4 0}$ undistorted signs. To insure continued accurate use of the written symbols, at the beginning of every session the subjects labeled 40 undistorted signs in which each of the handshapes was represented twice in random order.

Data reduction. Responses are tabulated in a 20 by 20 confusion matrix in which each stimulus appears at the head of a column and each response marks a row. Entries along the major 
diagonal are correct identifications. Off-diagonal entries are misidentifications. This full matrix is collapsed into a half matrix when confusionfrequencies are normalized. With a formula given by Shepard $(1962,1972$, pp. 67-113), a similarity score is calculated for each of the 190 possible nonidentical pairs of handshapes:

$$
S_{i j}=\left(f_{i j}+f_{j i}\right) /\left(f_{i i}+f_{j j}\right) \text {. }
$$

The similarity measure relating the $i^{\text {th }}$ and $j^{\text {th }}$ stimuli $\left(S_{i j}\right)$ is set equal to the sum of the frequencies of their confusions $\left(f_{i j}+f_{j j}\right)$ normalized by the sum of the frequencies of their correct identification $\left(f_{i i}+f_{j j}\right)$.

These similarity scores are entered into a program employing D'Andrade's (1978) U-statistic hierarchical clustering. This procedure provides a similarity structure for $\mathrm{K}$ elements from confusion data in which the $K$ elements are represented as the terminal points of a binary branching tree structure. A node in the tree is a point at which two branches diverge. The single node to which all branches converge is given a rank of one. Points of divergence after that are given higher rank orders until the terminal nodes, dominating single pairs of the $\mathrm{K}$ elements, are given the highest rank orders. The highest valued node level that dominates two terminal elements is a measure of the similarity of these elements, according to the tree. Larger node values indicate greater similarity. Besides constructing a tree from similarity scores, the program can also accept an existing tree structure as input. In this case, the program tests how well the nodes of the tree structure fit a set of similarity scores for all possible pairs (where order does not differentiate pairs) of the $\mathrm{K}$ elements that the tree structure is intended to represent.

Percent correct binary predictions is a measure of the goodness of fit of a tree structure to the similarity scores. The number of times the rank order predictions made by the tree match the rank order of similarity scores in the data equals the total number of correct predictions. This number divided by the total number of predictions made by the tree equals the percent correct binary predictions. Total number of predictions is calculated as:

$$
T=2 \cdot\{K ! /[3 ! \cdot(K-3) !]\} .
$$

For every possible combination of $\mathrm{K}$ elements taken three at a time, a binary tree makes two predictions about the order of similarity of pairs of those three elements and has nothing to say about the third possible prediction.

A simple example of confusions among four elements will help clarify the method of data analysis. The uncollapsed confusion matrix for four elements E, A, Y, and I appears in Table 1, part A. The collapsed matrix of similarity scores that enters into the clustering analysis appears in part $B$. The structure that is produced by the analysis to represent the similarity of elements $\mathrm{E}, \mathrm{A}$, $Y$, and $I$ to one another appears in part $C$. From the formula in part $D$, we can see that, for $K=4$ elements, the binary tree makes eight predictions about the similarity of the elements and has nothing to say about four additional predictions. The predictions made by the tree structure in part $\mathrm{C}$, and those predictions it fails to make, are listed in part E. Here we can see, for example, that the tree predicts that the pair EA will have a larger similarity score than AY; the highest valued node level dominating EA is 2, while that dominating $A Y$ is only 1 . This prediction is correct (as are the seven other predictions made by the tree), since the similarity score for EA, 2904, exceeds the score for AY, 2256. On the other hand, the tree makes no rank-order (binary) prediction about the similarity of AY and AI, for example; the highest valued node level dominating each pair is the same, 1 , and no rank-order prediction is possible. For this tree structure, the percent correct binary prediction is 100 . However, if one of the eight predictions made by the tree had been wrong, the percent correct binary predictions would have been lowered to $7 / 8$, or $87.5 \%$, correct.
Table 1

A Simple Example of Confusions Among Four Elements to Help Clarify the Method of Data Analysis

(A) Uncollapsed Confusion Matrix

$\begin{array}{crrrr} & \text { E } & \text { A } & \text { Y } & \text { I } \\ \text { E } & 207 & 61 & 39 & 35 \\ \text { A } & 36 & 127 & 26 & 24 \\ \text { Y } & 14 & 32 & 130 & 70 \\ \text { I } & 7 & 20 & 95 & 158\end{array}$

(B) Collapsed Matrix of Similarity Scores

$$
\begin{array}{llll}
\text { E } & \text { A } & \text { Y } & \text { I }
\end{array}
$$

E

$\begin{array}{lll}\text { A } & 2904 & \\ \text { Y } & 1572 & 2256\end{array}$

I $\quad 1150 \quad 1543 \quad 5729$

(C) Tree Structure Produced by the Clustering Analysis

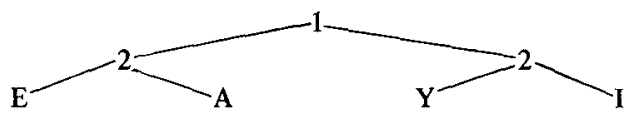

(D) Calculation of Total Number of Predictions About Similarity Made by the Tree Structure

$2 \times(4 ! /(3 ! \times(4-3) !))=8$ predictions

\begin{tabular}{|c|c|c|c|c|c|c|c|c|}
\hline \multicolumn{3}{|c|}{ Pair Names } & \multicolumn{3}{|c|}{ Node Levels } & \multicolumn{3}{|c|}{ Similarity Scores } \\
\hline $\mathrm{EA}$ & $>$ & EY & 2 & $>$ & 1 & 2904 & $>$ & 1572 \\
\hline EA & $>$ & $\mathrm{EI}$ & 2 & $>$ & 1 & 2904 & $>$ & 1150 \\
\hline EA & $>$ & AY & 2 & $>$ & 1 & 2904 & $>$ & 2256 \\
\hline EA & $>$ & AI & 2 & $>$ & 1 & 2904 & $>$ & 1543 \\
\hline YI & $>$ & YE & 2 & $>$ & 1 & 5729 & $>$ & 1572 \\
\hline YI & $>$ & IE & 2 & $>$ & 1. & 5729 & $>$ & 1150 \\
\hline YI & $>$ & YA & 2 & $>$ & 1 & 5729 & $>$ & 2256 \\
\hline YI & $>$ & IA & 2 & $>$ & 1 & 5729 & $>$ & 1543 \\
\hline AI & $?$ & EY & 1 & $?$ & 1 & 1543 & $?$ & 1572 \\
\hline EY & $?$ & EI & 1 & $?$ & 1 & 1572 & $?$ & 1150 \\
\hline EI & $?$ & AY & 1 & $?$ & 1 & 1150 & $?$ & 2256 \\
\hline AY & $?$ & AI & 1 & ? & 1 & 2256 & ? & 1543 \\
\hline
\end{tabular}

$1 \times(4 ! /(3 ! \times(4-3) !))=4$ nonpredictions

(E) Predictions and Nonpredictions Made by the Tree*

*Stated in terms of the pair names, in terms of the correspond. ing highest valued node level dominating the pair in the tree, and in terms of the corresponding similarity scores.

\section{Results and Discussion}

First, consider the consistency of confusion patterns without reference to a model. Signers and nonsigners were remarkably alike in their pattern of handshape confusions both within and between groups. The average intersubject reliability of confusion frequencies pooled over five sessions was quite high and identical within groups of signers and groups of nonsigners, $r=.84$. Consequently, confusion matrices were pooled over subjects and sessions within each group to produce a total of 405 observations per handshape. The full matrices of nonnormalized confusion frequencies for signers and nonsigners appear in Tables 2 and 3, respectively. ${ }^{2}$ These matrices are included for those who may wish to employ summary statistics different from those 
Table 2

Matrix of Handshape Confusion Frequencies for Signers

\begin{tabular}{|c|c|c|c|c|c|c|c|c|c|c|c|c|c|c|c|c|c|c|c|c|}
\hline & $X$ & bO & $\mathrm{O}$ & C & E & A & $\mathrm{Y}$ & I & 5 & 8 & $F$ & W & B & $\mathrm{V}$ & 3 & $\mathbf{R}$ & $\mathbf{H}$ & L & G & $\mathrm{K}$ \\
\hline $\mathrm{X}$ & 137 & 31 & 14 & 12 & 14 & 13 & 6 & 11 & 5 & 5 & 14 & 5 & 6 & 10 & 12 & 15 & 15 & 12 & 22 & 21 \\
\hline bO & 49 & 182 & 31 & 17 & 19 & 44 & 15 & 23 & 6 & 12 & 9 & 13 & 18 & 6 & 6 & 10 & 12 & 18 & 54 & 31 \\
\hline 0 & 7 & 27 & 186 & 38 & 19 & 31 & 20 & 6 & 5 & 5 & 11 & 2 & 9 & 2 & 0 & 3 & 4 & 6 & 5 & 1 \\
\hline C & 16 & 15 & 39 & 191 & 14 & 7 & 3 & 6 & 17 & 6 & 20 & 2 & 10 & 0 & 3 & 1 & 1 & 5 & 2 & 0 \\
\hline E & 10 & 21 & 63 & 36 & 207 & 61 & 39 & 35 & 2 & 2 & 7 & 9 & 19 & 7 & 0 & 5 & 14 & 6 & 10 & 2 \\
\hline A & 10 & 25 & 14 & 7 & 36 & 127 & 26 & 24 & 6 & 1 & 6 & 6 & 13 & 7 & 3 & 0 & 6 & 31 & 27 & 6 \\
\hline Y & 15 & 4 & 6 & 11 & 14 & 32 & 130 & 70 & 25 & 34 & 12 & 14 & 22 & 7 & 15 & 4 & 1 & 24 & 9 & 9 \\
\hline I & 3 & 4 & 17 & 6 & 7 & 20 & 95 & 158 & 4 & 12 & 8 & 14 & 8 & 1 & 16 & 3 & 3 & 12 & 7 & 5 \\
\hline 5 & 0 & 2 & 1 & 7 & 4 & 6 & 5 & 4 & 202 & 15 & 39 & 26 & 28 & 8 & 5 & 1 & 7 & 0 & 6 & 2 \\
\hline 8 & 13 & 6 & 4 & 5 & 7 & 3 & 14 & 13 & 35 & 177 & 37 & 40 & 22 & 9 & 18 & 10 & 15 & 11 & 8 & 5 \\
\hline$F$ & 5 & 8 & 5 & 8 & 9 & 1 & 7 & 10 & 14 & 28 & 139 & 14 & 22 & 6 & 4 & 3 & 11 & 1 & 5 & 2 \\
\hline W & 1 & 2 & 4 & 8 & 6 & 1 & 1 & 3 & 21 & 12 & 37 & 138 & 18 & 39 & 5 & 6 & 34 & 2 & 8 & 2 \\
\hline B & 2 & 3 & 4 & 20 & 7 & 6 & 1 & 7 & 9 & 1 & 5 & 23 & 173 & 0 & 4 & 1 & 5 & 5 & 1 & 1 \\
\hline V & 7 & 5 & 1 & 0 & 6 & 4 & 2 & 3 & 5 & 13 & 15 & 26 & 4 & 86 & 45 & 23 & 47 & 9 & 13 & 26 \\
\hline 3 & 9 & 3 & 2 & 0 & 1 & 1 & 4 & 1 & 14 & 19 & 15 & 20 & 6 & 30 & 90 & 16 & 35 & 18 & 16 & 14 \\
\hline $\mathbf{R}$ & 35 & 5 & 1 & 8 & 13 & 5 & 4 & 3 & 7 & 10 & 7 & 21 & 6 & 78 & 39 & 200 & 98 & 21 & 36 & 19 \\
\hline H & 7 & 1 & 2 & 3 & 4 & 3 & 3 & 1 & 2 & 5 & 6 & 11 & 4 & 23 & 36 & 35 & 70 & 8 & 12 & 15 \\
\hline L & 28 & 6 & 7 & 5 & 8 & 11 & 10 & 9 & 15 & 14 & 12 & 9 & 7 & 19 & 69 & 23 & 13 & 135 & 47 & 14 \\
\hline G & 26 & 34 & 4 & 6 & 7 & 24 & 11 & 15 & 4 & 11 & 7 & 6 & 5 & 14 & 13 & 15 & 14 & 51 & 99 & 47 \\
\hline K & 10 & 5 & 0 & 2 & 3 & 4 & 7 & 2 & 7 & 23 & 9 & 5 & 5 & 38 & 37 & 16 & 15 & 15 & 17 & 183 \\
\hline
\end{tabular}

Table 3

Matrix of Handshape Confusion Frequencies for Nonsigners

\begin{tabular}{|c|c|c|c|c|c|c|c|c|c|c|c|c|c|c|c|c|c|c|c|c|}
\hline & $X$ & bO & 0 & $\mathrm{C}$ & E & A & $\mathrm{Y}$ & I & 5 & 8 & F & W & B & V & 3 & $\mathbf{R}$ & $\mathbf{H}$ & L & G & $\mathrm{K}$ \\
\hline$X$ & 132 & 41 & 4 & 1 & 9 & 12 & 2 & 7 & 0 & 2 & 3 & 2 & 1 & 8 & 4 & 7 & 5 & 11 & 33 & 19 \\
\hline bO & 42 & 162 & 14 & 9 & 18 & 38 & 14 & 10 & 1 & 9 & 10 & 0 & 6 & 2 & 4 & 6 & 10 & 10 & 26 & 10 \\
\hline 0 & 17 & 30 & 192 & 31 & 63 & 43 & 24 & 24 & 5 & 3 & 20 & 4 & 10 & 2 & 3 & 1 & 7 & 10 & 5 & 1 \\
\hline C & 25 & 35 & 76 & 225 & 45 & 45 & 16 & 12 & 4 & 7 & 20 & 4 & 16 & 6 & 7 & 2 & 10 & 12 & 4 & 7 \\
\hline $\mathrm{E}$ & 29 & 15 & 44 & 30 & 125 & 68 & 30 & 20 & 11 & 4 & 6 & 17 & 12 & 8 & 3 & 8 & 9 & 9 & 22 & 7 \\
\hline A & 10 & 36 & 24 & 19 & 48 & 109 & 52 & 30 & 15 & 3 & 10 & 18 & 19 & 4 & 2 & 5 & 10 & 34 & 24 & 6 \\
\hline $\mathrm{Y}$ & 6 & 3 & 9 & 3 & 8 & 20 & 107 & 45 & 13 & 24 & 14 & 19 & 19 & 10 & 14 & 3 & 3 & 36 & 18 & 12 \\
\hline I & 15 & 11 & 18 & 8 & 26 & 24 & 103 & 194 & 3 & 28 & 14 & 21 & 21 & 7 & 12 & 4 & 1 & 13 & 11 & 12 \\
\hline 5 & 0 & 0 & 0 & 5 & 3 & 1 & 5 & 1 & 220 & 40 & 41 & 29 & 47 & 11 & 16 & 2 & 14 & 6 & 4 & 1 \\
\hline 8 & 2 & 2 & 5 & 13 & 4 & 3 & 5 & 5 & 16 & 101 & 19 & 13 & 6 & 8 & 11 & 3 & 4 & 8 & 5 & 30 \\
\hline F & 7 & 2 & 4 & 3 & 5 & 1 & 7 & 16 & 6 & 53 & 134 & 16 & 21 & 5 & 6 & 4 & 4 & 2 & 8 & 6 \\
\hline W & 1 & 0 & 1 & 3 & 3 & 2 & 5 & 2 & 23 & 22 & 32 & 137 & 17 & 70 & 10 & 24 & 42 & 2 & 6 & 10 \\
\hline B & 2 & 4 & 0 & 18 & 21 & 6 & 3 & 9 & 40 & 7 & 13 & 42 & 185 & 2 & 4 & 9 & 10 & 2 & 5 & 2 \\
\hline V & 11 & 3 & 2 & 6 & 3 & 2 & 2 & 3 & 11 & 23 & 14 & 33 & 2 & 100 & 55 & 26 & 48 & 11 & 16 & 31 \\
\hline 3 & 8 & 3 & 0 & 0 & 3 & 4 & 5 & 6 & 13 & 18 & 14 & 7 & 6 & 16 & 84 & 6 & 12 & 22 & 8 & 10 \\
\hline $\mathbf{R}$ & 8 & 3 & 1 & 1 & 2 & 3 & 1 & 3 & 5 & 6 & 7 & 13 & 1 & 36 & 15 & 114 & 57 & 17 & 22 & 15 \\
\hline H & 9 & 6 & 2 & 8 & 4 & 4 & 5 & 2 & 4 & 9 & 19 & 12 & 9 & 36 & 32 & 98 & 127 & 8 & 22 & 40 \\
\hline $\mathbf{L}$ & 15 & 6 & 3 & 3 & 4 & 1 & 10 & 7 & 11 & 14 & 10 & 4 & 0 & 12 & 65 & 15 & 7 & 119 & 34 & 17 \\
\hline G & 36 & 24 & 5 & 4 & 7 & 17 & 1 & 3 & 2 & 10 & 9 & 8 & 2 & 26 & 45 & 35 & 28 & 47 & 120 & 55 \\
\hline $\mathbf{K}$ & 13 & 4 & 0 & 0 & 4 & 2 & 6 & 5 & 2 & 21 & 10 & 6 & 4 & 21 & 24 & 18 & 11 & 11 & 12 & 110 \\
\hline
\end{tabular}

employed here. The correlation between signers' and nonsigners' similarity scores ${ }^{3}$ calculated from these pooled matrices was $r=.88$. Finally, the confusion pattern of both groups of subjects in this study was like that of the signers of Lane et al., even though their subjects, pooled, made only 54 observations per handshape. The similarity scores of signers and nonsigners correlated at $\mathrm{r}=.82$ and $\mathrm{r}=.81$, respectively, with the pooled similarity scores of Lane et al.'s signers.

The clustering program produced an empirical tree for each set of similarity scores accepted as input. Although empirical trees were produced for subjects on an individual basis, only statistics for grouped data appear here. The empirical tree for each group of subjects made 2,280 predictions about the rank order of members within similarity score pairs. For the signers' empirical tree, $80 \%$ of these predictions were correct and the node levels of the tree correlated at $r=.70$ with their similarity scores. The corresponding statistics for the nonsigners' empirical tree were $78 \%$ and $r=.71$. The empirical tree for the signers was modified to produce a revised binary model of handshape features. This revised model is referred to as LBB2 and is shown in Figure 2.

Both LBB2 and the signers' empirical tree displayed three major groups of handshapes: [+ compact], [+broad], and [-broad]. Minor modifications were made in the arrangement of handshapes within each of these three groups in the empirical tree to produce 


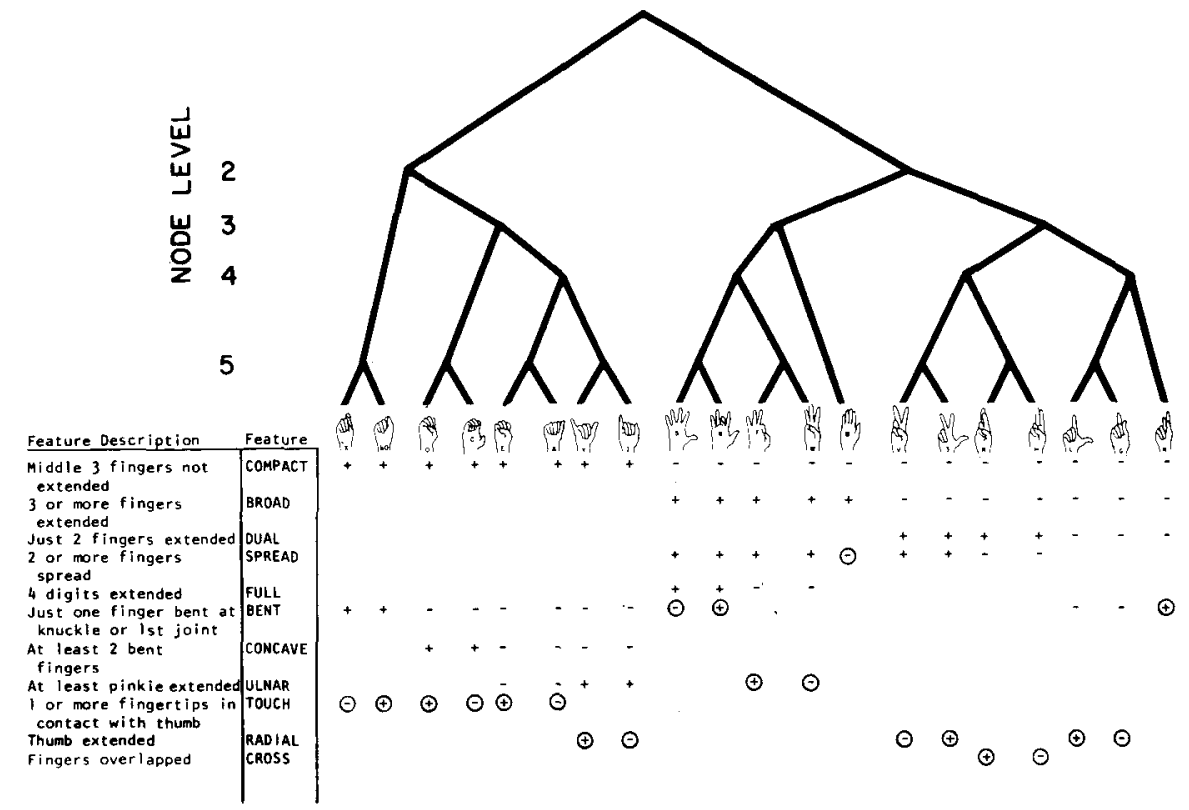

Figure 2. The revised version (LBB2) of the Lane et al. (1976) distinctive feature model for handshape in ASL.

LBB2. These modifications were made to allow an unequivocal criterion for assigning the + or - value of each feature to all handshapes while simultaneously maintaining the fit of the empirical tree to the similarity scores. Both objectives were accomplished. The criteria for feature assignment appear in Figure 2 under "Feature Description." They consider the hand to have five digits: four fingers and a thumb. LBB2 fits signers' similarity scores as well as their empirical tree, and it fits nonsigners' scores better than their empirical tree.

The node levels of LBB2 and the node levels of the Lane et al. model, LBB1, were read into the clustering program, and each was tested against three sets of similarity scores: the sets from the signers and nonsigners of this study and the set from the signers of Lane et al. Though based on relatively few observations per handshape and claiming only general independent support, impressively, LBB1 predicts the large corpora of signers' and nonsigners' confusions in this study equally well, and with the same degree of accuracy that the model predicts the confusions from which it was derived. Still, the revised model, LBB2, fits all three sets of similarity scores better than does LBB1. For the signers and nonsigners here, and for the signers of Lane et al., LBB2 makes correct predictions of similarity scores $80 \%, 82 \%$, and $78 \%$ of the time. The corresponding statistics from LBB1 are 76, 78, and 77. (Since each model makes 2,280 predictions, an increase of $1 \%$ correct predictions represents an increase of about 23 correct predictions.) Correlations between the node levels of LBB2 and the three sets of similarity scores were $r=.72, .71$, and .64 , respectively, whereas those from LBB1 were $.64, .69$, and .60 .

As well as being a modified version of the signers' empirical tree, LBB2 is a revision of LBB1 in structure and in the formal definition of handshape features. Although the difference between the models is small, the revisions discussed below were motivated by patterns in the empirical trees of signers' similarity scores that, on both an individual and a pooled basis, deviated consistently from LBB1.

All signers primarily confused $Y$ and I. Signers secondarily confused both members of the pair with the $[+$ compact $]$ hands (bO, X, O, C, E, and A) rather than with the [-broad] hands $(\mathrm{V}, \mathrm{R}, \mathrm{H}, \mathrm{L}$, G, 3, and K) as implied by LBB1. Consequently, $\mathrm{Y}$ and I handshapes were clustered with [+compact] hands in individual and pooled empirical trees and so appear in the [+compact] group in the revised model rather than in the [-broad] group. This is the major difference between LBB1 and LBB2 and is the reason for the redefinition of the [+ compact] feature from "no fingers extended" to "middle three digits not extended." A regrouping within the [+broad] hands was similarly motivated in the revised model. All signers saw 5 as more similar to $8, F$, and $\mathrm{W}$ than to B (in LBB1, 5B is the most similar pair). Finally, all signers saw 3 as more similar to $V, R$, and $H$ than to $L$ and $G$ (in LBB1, 3 is more similar to $L$ and $G$ than to $V, R$, and $H$ ). Because LBB2 is based on nearly eight times as many observations as LBB1, it is less the product of random error in its final form and therefore must be the more valid descriptor of handshape identification. Whether LBB2 is also an 
accurate description of handshape discrimination remains to be seen in Experiment 2.

Before proceding, a summary is in order. First, the features of the model (LBB2) seem to be functional in handshape identification, since they predict not only the handshape confusion patterns of the subjects in this study, but also the much smaller corpus of confusions from the Lane et al. study. Second, linguistic experience does not alter perception of visual features signifying linguistically contrastive handshapes. The model accurately predicts the confusion patterns of three groups of subjects with varying degrees of experience in identifying handshapes in a linguistic context. The nonsigners of this study had no experience, the native signers had maximal experience, and Lane et al.'s signers had had experience that was between these extremes.

Experiment 2 also tests the generality of the model, but in a different way. Earlier, we asked if the model would predict errors in handshape identification generated by various groups of subjects equally well. Now we ask if the model will predict errors in handshape perception generated by different tasks equally well. Experiment 2 employed a task to generate errors, which is paradigmatically different from handshape identification but which, like identification, has an outcome specifically predicted by the model. The task is handshape discrimination.

In Experiment 2, time compression was employed to degrade the discriminability of pairs of signs presented sequentially and thereby induce observers into error when they were asked to make "same-different" judgments about the handshape parameter of sign pair members. The results of Grosjean (1979) inspired the selection of time compression as a technique that might induce nonrandom errors in sign discrimination. He found that the movement of a sign was primarily responsible for its duration and that the mean duration of a sign at normal conversational speed was $360 \mathrm{msec}$, with a mean time between signs of $200 \mathrm{msec}$. For the discrimination task of Experiment 2, a sign was compressed to approximately half its normal duration, $166 \mathrm{msec}$, by filming only four selected points in the trajectory of the movement of the sign while it was being performed by a native signer. The time between presentation of members of a pair of signs was about one fifth the time between signs in normal conversation, $38 \mathrm{msec}$. These specific times were chosen for the sign duration and interstimulus interval (ISI) because they were expected to nonrandomly limit handshape information available for discrimination in two ways.

First, the 166-msec sign duration allows minimal time for observers to gather information about handshape identity while a sign is displayed. Averbach and Coriell (1961) found that perception of a complicated visual field was built up from information gathered during many fixations of the eye. The
$166 \mathrm{msec}$ duration is slightly below the estimated time for the eyes to change fixation, thereby allowing a single fixation per sign presentation.

Second, the $38 \mathrm{msec}$ ISI restricts the time for observers to encode information about the identity of the handshape of the first sign in a sequence, since the second sign onsets within the visual persistence of the first. Visual persistence is visual information that remains available to the subject after a briefly presented visual stimulus is terminated. It is phenomenally indistinguishable from the physical stimulus. It is generally thought of as an internal representation that reflects the physical parameters of the stimulus (Haber \& Standing, 1969) and that can be functional in encoding the stimulus. The duration of visual persistence can vary from only a few milliseconds (Efron, 1970) to as much as $250 \mathrm{msec}$ (Scharf \& Lefton, 1970; Sperling, 1960). It can be prolonged by at least two manipulations: (1) decreasing the stimulus duration (Briggs \& Kinsbourne, 1972; Efron, 1970); and (2) requiring subjects to note the of fset of a complex visual stimulus and report some property of the stimulus, as opposed to requiring subjects to note the offset without report (Erwin \& Hershensen, 1974; K. Green, 1980; Reef, Battison, \& Lane, Note 6).

When signs are compressed from their normal duration of $360 \mathrm{msec}$ to a duration of $166 \mathrm{msec}$, their estimated visual persistence, under a condition of no report, increases from 22 to $32 \mathrm{msec}$ (Briggs \& Kinsbourne, 1972). In the discrimination experiments, the ISI $(38 \mathrm{msec})$ between sign pair members just exceeds this estimated persistence. However, requiring "same-different" judgments of handshape pairs is expected to force a condition of report on the first sign in sequence such that its visual persistence exceeds the ISI and its encoding is therefore disrupted by the onset of the second sign. For signs presented in isolation, K. Green (1980) and Reef et al. (Note 6) found a significant increase in the visual persistence of signs when native signers were required to report the handshapes of the signs as opposed to when no report was required.

In Experiment 2, it was expected that subjects would be selectively impaired in handshape discrimination. For pairs of signs with different handshapes that share many features according to the model (e.g., $C$ and $O$, Figure 1), the information relevant to responding "different" is unlikely to have been extracted when the second sign occurs. Subjects will tend to respond "same" and the discrimination indices for these pairs will be low. For handshape pairs that share few features, the feature critical to responding "different" is likely to have been extracted from the first sign before encoding is disrupted by the second sign. Discrimination indices for these pairs will be high. Finally, if linguistic experience is not necessary to consistently use the same set of features across tasks commonly requiring handshape dif- 
ferentiation, the model will predict the rank order of handshape discrimination indices for nonsigners as well as it does for signers.

\section{EXPERIMENT 2}

\section{Method}

Subjects. The subjects were the same as those in Experiment 1. Stimuli and Apparatus. Eighteen pairs of handshapes (of the total possible 190 combinations) were chosen for the discrimination study. Each pair contained one of the six unmarked hands (see Battison, 1974) of ASL: O, A, C, B, G, and 5. Among other characteristics that distinguish this group: they are the only handshapes (with a few exceptions) that can serve as the basehand in nonsymmetrical signs; they comprise $69 \%$ of all entries in the Stokoe et al. (1965) Dictionary of American Sign Language; and they are acquired early in life by deaf native signers (Boyes-Braem, Note 7; Mclntyre, Note 8).

Treating each unmarked hand as a standard, three comparison hands were selected for high, intermediate, and low confusability according to the Lane et al. model. A pair of high confusability was dominated by a node level 6 in the model (the pair shares two or more nonredundant ${ }^{3}$ features); intermediate confusability was dominated by node level 4 (pair shares one or two features); and low confusability was dominated by node level 1 (pair shares one or no features). ${ }^{6}$ From the highest to the lowest level of confusability, comparisons were, to $\mathrm{O}: \mathrm{C}, \mathrm{X}$, and $\mathrm{B}$; to $\mathrm{G}: \mathrm{L}, \mathrm{H}$, and bO; to $\mathrm{A}$ : $\mathrm{E}, \mathrm{O}$, and $\mathrm{I}$; to $5: \mathrm{B}, \mathrm{F}$, and $\mathrm{X}$; to $\mathrm{B}: 5,8$, and $\mathrm{E}$; and to $\mathrm{C}$ : $O, X$, and 3 (see Figure 1 for the handshapes corresponding to these symbols and Table 4 for a full list of the comparisons to each handshape standard).

A session consisted of 432 trials. Each of the 18 handshape pairs was represented by 24 trials $(18 \times 24=432)$. The trials for each pair were divided into two trial types: 12 "same" trials, subdivided into 6 trials of one member of the pair presented twice and 6 trials of the other member presented twice, and 12 "different" trials, subdivided into 6 trials of member 1 followed by member 2, and 6 trials with the reverse order of presentation. On a trial, pair members were presented sequentially preceded by a warning stimulus. The hand did not remain stationary during presentation of each pair member. It appeared as part of a sign that is formationally permissible, but not meaningful, in ASL. Thus, a native signer, framed from the top of his head to his waist, performed each sign, and palm orientation, location, and movement varied from one pair member to the next. Across the 18 handshape pairs and across the two trial types within a pair, palm orientation, location, and movement varied over three values each (the same values used by Lane et al.) in counterbalanced fashion.'

All sign pairs were time compressed and presented on $16-\mathrm{mm}$ black-and-white film at, on the average, half the duration of signs in normal conversation (Grosjean, 1979). A single framing technique accomplished the time compression. As a native signer performed each sign, he segmented its trajectory of movement into four points: the start, the finish, and two positions equally spaced between the extremes. Each of these four points was photographed in one frame of film and the four frames comprised the sign. A finished trial consisted of 89 frames: a warning stimulus (the signer seated in a chair with his hands folded in his lap), four frames; a blank, four frames of black film; sign 1, four frames; the interstimulus interval, one frame of black film; sign 2, four frames; and the intertrial interval, 72 frames of black film. Played at normal speed, 24 frames/sec (Kodak Pageant 16-mm projector), the warning stimulus, blank, and each nonsense sign were $166 \mathrm{msec}$ each, the interstimulus interval was $38 \mathrm{msec}$, and the intertrial interval was $3 \mathrm{sec}$. The 432 trials were distributed randomly throughout the finished film.

The subjects viewed the film on a rear-projection screen through a tachistoscope (Scientific Prototype 320GB). The onset of each
Table 4

Discrimination Indexes for Handshape Standards and Their Comparisons

\begin{tabular}{|c|c|c|c|c|c|}
\hline \multirow{2}{*}{$\begin{array}{l}\text { Node } \\
\text { Level }\end{array}$} & \multirow{2}{*}{$\begin{array}{l}\text { Compar- } \\
\text { ison }\end{array}$} & \multicolumn{2}{|c|}{$\mathrm{d}^{\prime}$} & \multicolumn{2}{|c|}{$d_{s}$} \\
\hline & & NS & $S$ & NS & $\mathbf{S}$ \\
\hline & & & & & \\
\hline 6 & $\underline{C}$ & .73 & .89 & .27 & .52 \\
\hline 4 & $\underline{\bar{A}}$ & 1.12 & 1.37 & .51 & .90 \\
\hline 4 & $\overline{\mathbf{E}}$ & .26 & .43 & .13 & .22 \\
\hline 4 & $\mathrm{X}$ & 1.25 & 1.71 & .74 & 1.44 \\
\hline \multirow[t]{2}{*}{1} & $\underline{B}$ & 1.71 & 2.12 & .91 & 1.34 \\
\hline & & & & & \\
\hline 6 & $\mathbf{L}$ & .75 & 1.16 & .46 & .77 \\
\hline 4 & $\mathbf{H}$ & .62 & 1.29 & .42 & .80 \\
\hline \multirow[t]{2}{*}{1} & bO & 1.13 & 1.35 & .60 & .75 \\
\hline & & & & & \\
\hline 6 & $\mathrm{E}$ & 1.02 & 1.00 & .48 & .62 \\
\hline 4 & $\underline{\mathrm{O}}$ & 1.12 & 1.37 & .51 & .90 \\
\hline \multirow[t]{2}{*}{1} & $\overline{\mathrm{I}}$ & .89 & 1.07 & .60 & .78 \\
\hline & & & & & \\
\hline 6 & B & 1.95 & 1.65 & 1.09 & 1.07 \\
\hline 4 & $\bar{F}$ & 1.76 & 2.08 & .92 & 1.27 \\
\hline 4 & 8 & .91 & .80 & .53 & .57 \\
\hline \multirow[t]{2}{*}{1} & $X$ & 2.22 & 2.20 & 1.08 & 1.43 \\
\hline & & & & & \\
\hline 6 & 5 & 1.95 & 1.65 & 1.09 & 1.07 \\
\hline 4 & $\overline{8}$ & 2.39 & 2.46 & 1.14 & 1.63 \\
\hline 1 & E & 1.70 & 1.68 & .95 & 1.02 \\
\hline \multirow[t]{2}{*}{1} & $\underline{0}$ & 1.71 & 2.12 & .91 & 1.34 \\
\hline & & & & & \\
\hline 6 & $\underline{\mathrm{O}}$ & .73 & .89 & .27 & .52 \\
\hline 4 & $\bar{X}$ & 1.06 & 1.68 & .66 & 1.07 \\
\hline 1 & 3 & 1.76 & 2.45 & 1.08 & 1.57 \\
\hline
\end{tabular}

Note-NS = three ASL-naive hearing subjects; $S=$ three deaf native users of $A S L$.

trial triggered a photocell, which started a clock in a laboratory computer (Digital PDP-11). The subject's "same" or "different" keypress stopped the clock and the computer retained the reaction time along with the type of response in a data file.

Procedure. The subjects were well acquainted with the stimulus materials and apparatus before data collection began. The exact composition of a trial was explained and shown to them. They were told that there were two trial types for each of 18 handshape pairs, and types and pairs were demonstrated. The subjects were instructed only to compare handshapes of signs and to respond as quickly and accurately as possible with a "same" or "different" keypress.

Each subject participated in at least two practice sessions. In one, he was shown the film at varying speeds (Lafayette Sound Motion analyzer): 1, 2, 4, 6, 10, 12, and 24 frames/sec. Two successive correct responses resulted in an increased film speed, and one incorrect response decreased film speed (the subjects never scored less than $78 \%$ correct in this session). In other practice sessions, the film was run at normal speed ( $24 \mathrm{frames} / \mathrm{sec}$ ) and events proceeded as they would during the experiment. These sessions were intended to thoroughly familiarize subjects with the stimuli and with the format of a session, thereby optimizing discrimination performance, before data collection began.

Each subject participated in 10 experimental sessions (no more than two each day separated by at least $1 \mathrm{~h}$ ), resulting in 240 trials for each handshape pair. For half of these trials, the correct response was "same"; for the other half, the correct response was 
"different." In each new session, the film was begun at a different randomly determined location. The length of a session was approximately $1 \mathrm{~h}$, including a 15 -min break after a variable number of trials.

Data reduction. For each subject, two measures of discriminability were calculated for each of the 18 handshape pairs, one based on "same"', "different" responses, $d$ " and the other based on reaction times, $d_{s}$. For at least the signers, both discrimination indices were expected to decrease as handshape pairs displayed higher similarity scores and higher node levels according to either binary model. $\mathrm{d}^{\prime}$ is calculated as the $\mathrm{Z}$ score of the probability of a hit minus the $Z$ score of the probability of a false alarm (Green \& Swets, 1974). Here a hit is responding "different" when, in fact, the pair of signs displayed different handshapes. A false alarm is responding "different"' when the pair displayed the same handshape.

In calculating $\mathrm{d}_{s}$, reaction times were used as rating responses (Emmerich, Gray, Watson, \& Tanis, 1972; D. M. Green \& Swets, 1974, pp, 101-103) indicating the subjects' certainty that a "same", or "different" response had been correct after every trial. Shorter times indicate greater confidence. In this experiment, reaction times for each of the 18 handshape pairs were separated into those from noise (i.e., "same") trials and those from signal-plus-noise (i.e., "different") trials. Times from the noise trials were sorted into $\mathbf{4 0}$ bins ordered along a scale from the shortest to the longest "false alarm" reaction times through the longest to the shortest "correct rejection" times. The corresponding scale for the signalplus-noise trials was ordered from the shortest to longest "hit" reaction times through the longest to shortest "miss" times (A "correct rejection" is responding "same," given that the handshapes of the signs were the same; a "miss" is responding "same," given that the handshapes of the signs were different.) Along each of these two scales, cumulative probabilities were computed; these are probabilities that a reaction time falls at or below the upper limit of each successive bin. The $\mathbf{4 0}$ pairs of cumulative probabilities were transformed into $\mathrm{Z}$ scores. These pairs were used to plot a ROC curve in linear coordinates which was fit by a least squares criterion. Twice the value of $x$, for which the two equations $y=m x+b$ (the ROC curve) and $y=-x$ (the minor diagonal of the ROC square) are equal, is $\mathrm{d}_{\mathbf{s}}$ for a handshape pair.

\section{Results and Discussion}

Without reference to a model, consider performance between groups of subjects. Signers were more accurate, faster, and less variable than nonsigners in discriminating handshape differences. Signers' $d^{\prime}$ and $d_{s}$ values were significantly higher than the corresponding values for nonsigners $[\mathrm{t}(34)=3.54$ and 7.11 for $d^{\prime}$ and $d_{s}$, respectively, $p<.001$, one-tailed]. Reflecting the $d_{s}$ finding, signers' reaction times (mean, $485 \mathrm{msec}$ ) were significantly shorter than nonsigners' reaction times [mean, 509 msec; $\mathrm{t}(3)=2.43, \mathrm{p}<.01$, one-tailed]. Furthermore, the variability of signers' reaction times $(S D=181 \mathrm{msec})$ was significantly smaller than that of nonsigners $(\mathrm{SD}=215 \mathrm{msec} ; \mathrm{F}=$ $4.79, \mathrm{p}<.01)$.

Within a group, signers were more uniform in their patterns of discrimination indices. The average intersubject reliability of $\mathrm{d}^{\prime}$ and $\mathrm{d}_{\mathrm{s}}$ scores was higher for signers than for nonsigners: for signers' $d^{\prime}, r=.76$, and signers' $d_{s}, r=.80$; for nonsigners' $d^{\prime}, r=.61$, and nonsigners $d_{s}, r=.64$. Within each group of subjects, the procedure by which reaction times were transformed into indices of discriminiability was vali- dated. There was a high correlation between patterns of pooled $d^{\prime}$ and $d_{s}$ scores (see Table 4): $r=.99$ for signers and $r=.96$ for nonsigners. This corroborates the findings of other studies (Emmerich et al., 1972; M. Green, Terman, \& Terman, 1979) that, in addition to latencies accompanying correct responses, latencies accompanying errors are valuable in assessing a subject's capacity to make a particular stimulus discrimination. [The convergence of discrimination indices, from binary decisions $\left(d^{\prime}\right)$ and from latencies of binary decisions $\left(d_{s}\right)$, on a single pattern of handshape discriminability bolsters confidence in using that pattern to choose between models of feature processing later in the General Discussion section.] Since the correlations between $d_{s}$ and $d^{\prime}$ are so high, henceforth we will speak only of $d^{\prime}$ values. It should be understood that what holds for $\mathrm{d}^{\prime}$ also holds for $d_{s}$.

Looking across identification and discrimination tasks, first consider that in discrimination, as in identification, the relative saliency of pairs of handshapes for signers and nonsigners was essentially the same. The correlation between deaf and hearing $\mathrm{d}^{\prime}$ values was high, $r=.88$. Therefore, as we would expect, identification performance predicted discrimination performance for both groups of subjects. The $d^{\prime}$ values were significantly correlated $(p<.01)$ with the normalized confusion frequencies of their respective handshape pairs at $\mathrm{RHO}=-.79$ for signers and -.71 for nonsigners. Finally, also as expected, the revised binary model was more accurate than the original model in predicting the discrimination performance of both groups of subjects. Correlations between LBB2 nodes and $d^{\prime}$ values ${ }^{8}$ were RHO $=-.83$ and -.63 for signers and nonsigners, respectively, while the same correlations for LBB1 nodes were - .73 and -.26 .

In summary, subjects use the same set of features to identify and discriminate handshapes despite very different histories in processing handshape information in a linguistic context. The revised binary model (LBB2) represents this set of features well. In the studies reported here, linguistic experience is manifest in the performance of signers only to the extent that they are more accurate, faster, and less variable than nonsigners in handshape discrimination.

\section{GENERAL DISCUSSION}

\section{A Continuous Model}

A binary account of handshape primes perceived as discrete bundles of LBB2 distinctive features is consistent with the data. But this is not the only consistent account. A nonbinary account of handshape primes continuously perceived in (an as yet unmeasured) two-dimensional space is an alternative model. Thus, handshapes, like vowels in spoken language, might be characterized in binary fashion for some 
2 DIMENSIONAL MODEL OF HANDSHAPE SIMILARITY STRUCTURE

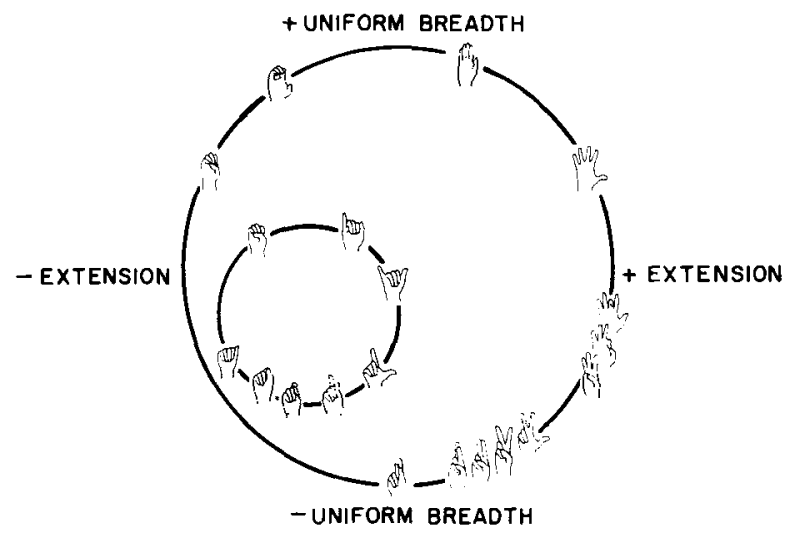

Figure 3. Two-dimensional model of handshape similarity in which two features of handshape structure vary continuously.

purposes, but, in fact, they differ continuously along physically measurable dimensions that predict an observer's confusions among members of the phonetic set.

Figure 3 is a two-dimensional continuous model of handshape similarity that is derived from twodimensional scaling (Kruskal, 1964a, 1964b; Shepard, 1962) of signers' handshape confusions. ${ }^{9}$ This model suggests that there are two continuously variable features that play a major role in determining the similarity of handshapes. One feature is [extension]. In terms of articulation, as handshapes decrease in [extension], digits fold toward the palm of the hand; visually (as the hand is viewed with the palm toward the observer), the perimeter of the image of the hand shrinks. The other feature is [uniform breadth]. In terms of articulation, as handshapes decrease in [uniform breadth], motor commands to the five digits are more various and, visually, the image of the hand appears less symmetrical about the middle digit.

This model fits all available sets of identification and discrimination data well, and at least as well as the binary model. The correlation between similarity scores and their corresponding interpoint distances (IPDs) in the model is $\mathrm{r}=-.79,-.72$, and -.65 for signers, nonsigners, and Lane et al.'s signers, respectively. (The analogous statistics for LBB2 were $r=.72$, .71 , and .64 , respectively.) The correlation between $\mathrm{d}^{\prime}$ values and corresponding IPDs is $\mathrm{RHO}=.80$ and .64 for signers and nonsigners, respectively. (The correlation between $d^{\prime}$ and number of shared nonredundant features according to $\mathrm{LBB} 2$ is $\mathrm{RHO}=-.83$ and -.63 , respectively).

\section{Binary or Continuously Variable Features?}

Which is the better descriptor of handshape identification and discrimination: binary features or con- tinuously variable features? It is not easy to demonstrate. The binary model makes ordinal predictions about handshape confusability, while the continuous model makes interval scale predictions. They are not statistically comparable in any direct way. Therefore, it is difficult to conduct a straight forward test for a significant difference between the models in their accuracy of predicting a set of similarity scores. Measures of goodness of fit for binary and continuous representations (percent correct binary predictions and stress, respectively) are not of much help in deciding between models, since they, too, are not statistically comparable in any direct way.

It is possible to treat the interpoint distances of the continuous model like nodes of a binary model and to employ them as ordinal scale predictors of similarity scores. We can calculate a rank-order correlation coefficient and see how predictors from continuous and binary models compare. RHO between interpoint distances of the continuous model and the similarity scores of signers, nonsigners, and Lane et al.'s signers were: $-.81,-.73$, and -.60 , respectively. RHO between the nodes of the best-fitting binary model, LBB2, and the same sets of similarity. scores were: $-.47,-.49$, and -.40 , respectively. Clearly, without tests for a significant difference between corresponding vaues of RHO, the continuous model is the better predictor of each set of data. Still, RHO may not be the best statistic to use to decide between the models, since there are many more ties in the rank order of node levels than there are in the rank order of interpoint distances. Many ties in ranks lower RHO considerably. While the Pearson correlation coefficient has been used (here and in Lane et al.) to make ordinal comparisons between the descriptive adequacy of alternative binary models, its utility in choosing between binary and continuous models is questionable. The statistic requires a normal distribution of the two correlated variables, and node levels are certainly not normally distributed. Given the aforementioned statistical problems, it is best to examine the handshape discrimination data to decide between models.

Using redundant features, it is possible to derive interval scale metrics of discriminability from the binary model to directly compare its descriptive adequacy and predictive validity with those of the continuous model. ${ }^{10}$ To derive the metrics, fill in the feature matrix at the bottom of Figure 2, count the total number of features by which members of a pair of handshapes differ, and use this number as the metric of the pair's discriminability. The corresponding metric of a pair's discriminability in the continuous model is the distance between pair members in twodimensional Euclidian space.

Descriptive adequacy refers to how well a model's metric of discriminability coincides with a pattern of $d^{\prime}$ values obtained. Predictive validity refers to how 
well a model's metric coincides with a pattern of $d^{\prime}$ calculated from obtained values using a combinatorial rule unique to the model. With both descriptive adequacy and predictive validity, interest centers on which model accounts for the larger percentage of variance in the pattern of $d^{\prime}$ values.

With reference to descriptive adequacy, the continuous model is clearly preferable to the binary model for the signers' data. For signers, the IPDs of the continuous model account for $72 \%(r=.85)$ of the variance in obtained $\mathrm{d}^{\prime}$ values, while total number of features different according to the binary model accounts for only $59 \%(r=.77)$ of the variance. The nonsigners' data do not permit preference for a model. Continuous and binary models each account for about half the variance in obtained $\mathrm{d}^{\prime}$ values: $49 \%(r=.70)$ and $55 \%(r=.74)$, respectively.

With reference to predictive validity, the continuous model is again clearly preferable to the binary model, this time for both signers' and nonsigners' data. Preference for the continuous model in the area of predictive validity arose as follows.

Each model specifies a different rule for combining two known $d^{\prime}$ values to obtain an unknown value when the two known values are for two handshape pairs that have a member in common, for example, the two pairs $\mathrm{XO}$ and $\mathrm{OA}$. The binary model requires the additive combination of the two known values to obtain the unknown value, since the metric of discriminability is the total number of features by which members of a pair of handshapes differ:

$$
d^{\prime} X^{-} d^{\prime}{ }_{O A}=d^{\prime} X A^{\prime}
$$

The pair XO differs by three features, [bent], [concave], and [touch], while the pair OA differs on only two of these three features, [concave] and [touch]. To find the predicted value for the pair XA, which differ only on the feature [bent], it is necessary to subtract. For signers, $d^{\prime} \mathrm{XO}=1.71$ and $\mathrm{d}^{\prime} \mathrm{OA}=1.37$ so that the predicted $\mathrm{d}^{\prime} \mathrm{XA}=1.71-1.37=.34$. The predicted value is paired with its corresponding metric from the model, 1 (the number of features by which $\mathrm{X}$ and $\mathrm{A}$ differ), and is entered into the correlation of binary model metrics with predicted d'.

The continuous model requires combination of the two known values using an angle from the model (Figure 3) and the law of cosines to obtain the unknown value, since the metric of discriminability is Euclidian distance in two dimensional space between members of a handshape pair:

$$
\begin{array}{r}
\sqrt{\left(d^{\prime} X O\right)^{2}+\left(d^{\prime} O A\right)^{2}-2\left(d^{\prime} X O\right)\left(d^{\prime} O A\right) \cos (L X O A)} \\
=d^{\prime} x A .
\end{array}
$$

The pairs $\mathrm{XO}$ and $\mathrm{OA}$ each have corresponding vectors $^{11}$ in Figure 3 with a common endpoint, $O$.
These vectors form the angle XOA and also form two sides of a triangle. To find the predicted value for the pair XA, the vector length forming the third side of the triangle, it is necessary to use angle XOA and the law of cosines:

$$
\sqrt{(1.71)^{2}+(1.37)^{2}-2(1.71)(1.37)(.98)}=.46 .
$$

As before, the predicted value is paired with its corresponding metric from the model, 1077 (the distance between points corresponding to handshapes $\mathrm{X}$ and $A$ in Figure 3), and enters into the correlation of continuous model metrics with predicted $\mathrm{d}^{\prime}$.

From the $d^{\prime}$ values obtained for the 18 pairs of handshapes, 38 predictions of $\mathrm{d}^{\prime}$ are possible. That is, of the $18 \times 18$ possible combinations of two handshape pairs, 38 combinations share a handshape and so make it possible to predict a $d^{\prime}$ value for the unshared members of the two pairs. Of these 38 predictions, there are 32 made by the continuous model which are also made by the binary model. ${ }^{12}$ These 32 entered into the tests of the predictive validity of each model.

The continuous model is clearly preferable to the binary model in accounting for a larger percentage of the variance in the predicted scores. The IPDs of the continuous model account for $76 \%(r=.87)$ and $74 \%$ $(r=.86)$ of the variance in the $d^{\prime}$ values predicted according to the model's combinatorial rule for signers and nonsigners, respectively. The number of features different according to the binary model accounts for only $56 \%(r=.75)$ and $61 \%(r=.78)$ of the variance in its predicted values for signers and nonsigners, respectively. If pairs involving the handshape, B, are excluded from the tests of predictive validity, we are left with 24 predicted d' values. With the continuous model, the percentage of variation accounted for in predicted $d^{\prime}$ values remains unchanged. With the binary model, the percentage of variation accounted for increases, from $56 \%$ to $77 \%(r=.88)$ in the case of signers and from $61 \%$ to $85 \%(r=.92)$ in the case of nonsigners.

B is simply one of several handshapes whose similarity to other handshapes is only partially represented by the binary model. Thus, the model is a more accurate descriptor of handshape similarity when comparisons involving $B$ are excluded from the tests of predictive validity. LBB2 separates handshapes into three major groups: [+compact], [-compact, +broad], and [-compact,-broad]. Generally, handshapes from different major groups differ on more features and should be confused less than handshapes from the same major group. However, this does not hold for at least six handshapes: $\mathrm{B}, \mathrm{bO}, \mathrm{X}$, $\mathrm{G}, \mathrm{Y}$, and L. In identification, each of these is highly confused with at least two other handshapes from another major group in addition to those within its group. Since the model fails to represent this cross- 
Table 5

Six of the 18 Handshape Pairs From the Discrimination Experiment and Their Obtained $d^{\prime}$ Values

\begin{tabular}{ccccc}
\hline \multicolumn{2}{c}{$\mathrm{d}^{\prime}$} & & Handshape & \\
Nonsigners & Signers & $\begin{array}{c}\text { Pair } \\
\text { IPD }\end{array}$ & LBB2* \\
\hline .91 & .80 & 85 & 2316 & 1 \\
1.95 & 1.65 & $5 B$ & 2570 & 2 \\
2.39 & 2.46 & $8 B$ & 4560 & 3 \\
1.70 & 1.68 & BE & 4407 & 5 \\
.26 & .43 & EO & 1310 & 1 \\
1.71 & 2.12 & BO & 4528 & 6 \\
\hline
\end{tabular}

Note-The metric of discriminability from the continuous model (IPD) is consistent with the pattern of obtained d'values, but the metric from the binary model (LBB2) is clearly not consistent. $\quad$ Number of features different from $L B B 2: 1=$ bent; $2=$ spread, radial; $3=$ spread, bent, radial; $5=$ compact, broad, full, ulnar, touch; $1=$ concave; $6=$ compact, broad, full, concave, ulnar, touch.

category similarity, it incurs a large number of incorrect predictions (half of the incorrect predictions it makes) for those six handshapes.

When it comes to describing cross-category discriminability relative to within-category discriminability, the model is similarly impaired, particularly for handshape comparisons involving $B$. To illustrate, $B$ is [-compact, + broad], while the handshapes $E$ and $O$ are both from a different major group [+compact]. According to the binary model, $\mathrm{B}$ and $\mathrm{E}$ differ by five features while B and $O$ differ by six features. Both pairs of handshapes should yield substantially higher discrimination indices than the pair B8, since 8 is from the same major group as B (i.e., [-compact, +broad]) and 8 differs from B by only three features. However, the opposite occurs. B8 yields a higher discrimination index than either BE or BO (see Table 5). This outcome is consistent with the continuous, but not the binary, model. ${ }^{13}$

Handshapes are perceived in continuous fashion along [compact] and [broad] feature dimensions. Respectively, these two dimensions correspond roughly to [extension] and [uniform breadth] in the continuous model. Subjects must have access to information about degrees [compactness] and [breadth] and so perceive $B$ as having about the same degree of visual similarity to $\mathrm{O}$ and $\mathrm{E}$ as it does to 8 .

\section{A Prototype Model of Continuously Variable Handshape Features}

The continuous model of visual features proposed here for perception of handshape in ASL is consistent with continuous models of acoustic features advanced by Oden and Massaro (1978) and Repp (1977) for perception of consonants in English. Their models, in turn, are based on models of complex pattern recognition proposed by Lockhead $(1970,1972)$ and Selfridge (1959).

Both Oden and Massaro (1978) and Repp (1977) found, as was found here, that response patterns in the identification of linguistic segments were better explained by perception of continuously variable features than by binary ones. This led them to advance models of segment identification in which prototypes of speech segments are stored in long-term memory. In both Oden and Massaro's "fuzzy logical" model and Repp's "prototype" model, a stored prototype specifies the ideal acoustic feature values that a perceived acoustic stimulus would display if it were to be identified as an instance of a specific speech segment. The identification process occurs in at least three operations, beginning with a nonbinary determination of the value at which each feature is present in the acoustic stimulus and ending with a decision as to which stored prototype matches the stimulus best. The models differ in the type of mathematical function they employ to describe the intermediate operation-the integration of feature values to determine how well the acoustic stimulus matches each of several candidate prototypes. Because Repp uses a Euclidian distance function to describe the combined influence of features on prototype matching, as is intended here, we will discuss his model further.

A prototype model of continuously variable features in the perception of handshape is directly analogous to Repp's prototype model of continuously variable features in the perception of speech segments. The coordinates of handshapes in Figure 3 are considered to specify the loci of their corresponding prototypes in two-dimensional Euclidian space. The features [extension] and [uniform breadth] then correspond to the dimensions of the perceptual space relevant to prototype activation. The following points paraphrase Repp's (1977, p. 39) description of his model so that it describes the identification process of a visual stimulus, that is, a handshape: (1) Visual processing leads to a mapping of the visual information into the multidimensional space. (2) In this perceptual space, the stimulus leads to activation of the prototypes in its vicinity. The various degrees of activation are inversely and probably nonlinearly related to the Euclidian distance of each prototype from the stimulus. (3) A probabilistic decision process selects the prototype with the largest activation value as the percept and response.

Elaborating the prototype model of handshape, we expect that, under conditions of normal viewing, ASL handshapes map onto their respective prototypes with relatively little variability. A distortion procedure, such as masking the visual stimuli with noise, increases the variability of their mapping into the multidimensional space. During handshape identification, a degraded visual stimulus maps, on the average, farther away from its "correct" prototype than it normally would, so that prototypes in the vicinity are also activated by the degraded visual stimulus more than they normally would be. These other prototypes are given as misidentification of the degraded stimulus 
with a frequency that is directly proportional to their respective activation level and, therefore, inversely proportional to their Euclidian distance from the "correct" prototype. Thus, normalized confusion frequencies map the distance of prototypes relative to one another along the continuously variable feature dimensions germane to handshape identification.

In the discrimination experiment, time compression also increases the variability of two successive stimuli mapping onto their "correct" prototypes. The closer the two handshape prototypes are in the multidimensional space, the greater the probability that their two respective degraded stimuli will activate only one of the prototypes to the same degree during a different trial and that an incorrect "same" response will be made. Thus, the discrimination experiment should, and does, confirm the mapping of the handshape prototypes in the continuously variable feature space obtained from the identification experiment.

Finally, in contrast with the prototype model for consonants, the prototype model for handshape has no physically measurable dimensions specified that parallel its featural dimensions. The appropriate physical dimensions underlying [extension] and [uniform breadth] are unknown. (If the physical dimensions were known, we might name and define the featural dimensions differently.) However, continuous models of the type depicted in Figure 3 are expected to be useful in the search for the appropriate physical dimensions. Klein, Plomp, and Pols (1970), Pols, Tramp, and Plomp (1973), and Shepard (1972) have all found the multidimensional scalings of vowel confusions in two-dimensional Euclidian space (where features vary continuously, as Ladefoged, 1975, suggests) correlate very well with the first and second formant frequencies of the vowels. In that a continuous model of handshape perception in two dimensions accurately describes the identification and discrimination performances of two groups of subjects with disparate histories of handshape perception, it should predict the physical dimensions structuring handshape similarity.

The spatial frequency domain could be the first place to look for physical correlates of handshape feature dimensions. Campbell (1974) and Ginsberg (1971), among others, have given evidence for the existence of channels of spatial frequency analysis in the human visual system functional in the perception of visual form. The psychological similarity of handshapes could be a function of their spatial frequency composition. Research is currently directed toward investigating that possibility.

\section{CONCLUSION}

In sign, as in spoken, language, segments are perceived by means of distinctive features. Linguistic experience does not alter the visual features germane to handshape perception, since subjects with very different backgrounds in processing handshape information in a linguistic context exhibited very similar patterns of identification and discrimination performance. In like manner, Poizner and Lane (1978) found that linguistic experience did not alter the visual cues germane to perception of location in ASL.

Finally, a model of continuously variable visual distinctive features is more consistent with the available sets of handshape identification and discrimination data than is a binary model. This continuous model is directly analogous to the prototype model of continuously variable acoustic distinctive features advanced by Repp (1977) for identification of speech segments. Thus, the same process of complex pattern recognition seems to extend across the linguistic experience of subjects and the sensory modality of stimuli.

\section{REFERENCE NOTES}

1. Grosjean, F., Teuber, H., \& Lane, H. When is a sign a sign? The on-line processing of gated signs in American Sign Language. Unpublished manuscript, Northeastern University, 1979.

2. Stokoe, W. Sign language structure: An outline of the visual communication system of the American deaf (Studies in Linguistics, Occasional Papers, No. 8). Norman: University of Oklahoma, 1960.

3. Trager, G. L., \& Smith, H. An outline of English structure (Studies in Linguistics, Occasional Papers, No. 3). Norman: University of Oklahoma, 1951.

4. Nooteboom, S. G. Some regularities in phonemic speech errors (Onderzack Annual Progress Report 2). Eindhoven, The Netherlands: Institut voor Perceptie, 1967.

5. Newkirk, D., Pederson, C., \& Bellugi, U. Interference between sequentially produced signs. Unpublished manuscript, Salk Institute, La Jolla, Calif., 1975.

6. Reef, S., Battison, R., \& Lane, H. Visual persistence of handshapes in American Sign Language: An exploratory study. Unpublished manuscript, Northeastern University, 1977.

7. Boyes-Braem, P. The acquisition of handshape in American Sign Language. Unpublished manuscript, Salk Institute, La Jolla, Calif., 1973.

8. McIntyre, M. A modified model for the description of language acquisition in a deaf child. Unpublished manuscript, Department of Linguistics, California State University, Northridge, 1974.

9. Kruskal, J. B., \& Carmone, F. How to use M-D Scale (version 5-M) and other useful information. Unpublished manuscript, Bell Telephone Laboratories, Murray Hill, N.J., 1973.

\section{REFERENCES}

Abramson, A. S., \& Lisker, L. Discriminability along the voicing continuum: Cross-language tests. In Proceedings of the Sixth International Congress of Phonetic Science. Prague: Academia, 1970.

Averbach, M., \& Coriell, A. S. Short-term memory in vision. Bell System Technical Journal, 1961, 40, 309-328.

Battison, R. Phonological deletion in American Sign Language. Sign Language Studies, 1974, 5, 1-19.

Battison, R. Lexical borrowing in American Sign Language. Silver Spring, Md: Linstock Press, 1978.

Bellugi, U., Klima, E., \& Siple, P. Remembering in signs. Cognition, 1975, 3, 93-125.

Bellugi, U., \& Siple, P. Remembering with and without words. In F. Bresson (Ed.), Current problems in psycholinguistics. Paris: C.N.R.S., 1974. 
Briggs, G. G., \& Kinsbourne, M. Visual persistence as measured by reaction time. Quarterly Journal of Experimental Psychology, 1972, 24, 318-325.

Campeell, F. W. The transmission of spatial information through the visual system. In F. O. Schmitt \& F. G. Worden (Eds.), The neurosciences. Cambridge, Mass: M.I.T. Press, 1974.

Chomsкy, N., \& Halle, M. The sound pattern of English. New York: Harper \& Row, 1968.

ConRAD, R. An association between memory errors and errors due to acoustic masking of speech. Nature, 1962, 193, 1314-1315.

Conrad, R. Acoustic confusions in immediate memory. British Journal of Psychology, 1964, 55, 75-84.

D'Andrade, R. G. U-statistic hierarchical clustering. Psychometrika, 1978, 43, 59-67.

EFron, K. Effects of stimulus duration on perceptual onset and offset latencies. Perception \& Psychophysics, 1970, 8, 231-234.

Eımas, P. D., \& Corbit, J. D. Selective adaptation of linguistic feature detectors. Cognitive Psychology, 1973, 4, 99-109.

Emmerich, D. S., Gray, J. L., Watson, C. S., \& Tanis, D. C. Response latency, confidence, and ROCs in auditory signal detection. Perception \& Psychophysics, 1972, 11, 65-72.

Erwin, D. E., \& Hershenson, M. Functional characteristics of visual persistence predicted by a two factor theory of backward masking. Journal of Experimental Psychology, 1974, 103, 249-254.

Frishberg, N. Arbitrariness and iconicity: Historical change in American Sign Language. Language, 1975, 51, 696-719.

Fromкin, V. A. The non-anomalous nature of anomalous utterances. Language, 1971, 47, 27-52.

GinsBerg, A. P. Psychological correlates of a model of the human visual system. Masters thesis, Air Force Institute of Technology, Wright-Patterson AFB, 1971.

Goto, $H$. Auditory perception by normal Japanese adults of the sounds "l" or "r." Neuropsychologia, 1971, 9, 317-323.

Green, D. M., \& Swets, J. A. Signal detection theory and psychophysics. Huntington, N.Y: Kreiger, 1974.

Green, K. Visual persistence of moving handshapes in American Sign Language. Unpublished Masters thesis, Northeastern University, 1980.

Green, M., Terman, M., \& Terman, J. Comparison of yes-no and latency measures of auditory intensity discrimination. Journal of the Experimental Analysis of Behavior, 1979, 32, 363-372.

Grosjean, F. A study of timing in a manual and a spoken language: American Sign Language and English. Journal of Psycholinguistic Research, 1979, 8, 379-405.

Haber, R. N., \& Standing, L. G. Direct measures of short term visual storage. Quarterly Journal of Experimental Psychology, $1969,21,43-56$.

Klein, W., Plomp, R., \& Pols, L. Vowel spectra, vowel spaces and vowel identification. Journal of the Acoustical Society of America, 1970, 48, 999-1009.

Klima, E., \& Bellugi, U. The signs of language. Cambridge: Harvard University Press, 1979.

Kruskal, J. B. Multidimensional scaling by optimizing goodness of fit to nonmetric hypothesis. Psychometrika, 1964, 29, 1-27. (a)

Kruskal, J. B. Nonmetric multidimensional scaling: A numerical method. Psychometrika, 1964, 29, 115-129. (b)

LAdefoged, P. A course in phonetics. New York: Harcourt Brace Jovanovich, 1975.

Lane, H., Boyes-Braem, P., \& Bellugi, U. Preliminaries to a distinctive feature analysis of handshape in American Sign Language. Cognitive Psychology. 1976, 8, 263-289.

Liberman, A., Cooper, F. S., Shankweiler, D. P., \& Studdert-Kennedy, M. Perception of the speech code. Psychological Review, 1967, 74, 431-461.

LockHEAD, G. R. Identification and the form of multidimensional discrimination space. Journal of Experimental Psychology, $1970,85,1-10$.
Lockhe ad, G. R. Processing dimensional stimuli: A note. Psychological Review, 1972, 79, 410-419.

MACKAY, D. G. Spoonerisms: The structure of errors in the serial order of speech. Neuropsychologia, 1970, 8, 323-350.

Miller, G. A., \& Nicely, P. E. An analysis of perceptual confusions among some English consonants. Journal of the Acoustical Society of America, 1955, 27, 339-352.

Miyawaki, K., Strange, W., Verbrugge, R. R., Liberman, A. M., Jenkins, J. J., \& Fujimura, $O$. An effect of linguistic experience: The discrimination of [r] and [l] by native speakers of Japanese and English. Perception \& Psychophysics, 1975, 18, 331-340.

Norman, D. A. (Ed.). Models of human memory. New York: Academic Press, 1970.

Norman, D. A. Memory and attention. New York: Wiley, 1976.

ODEN, G. C., \& MASSARo, D. W. Integration of featural information in speech perception. Psychological Review, 1978, 85, 172-191.

Parker, F. Distinctive features and acoustic cues. Journal of the Acoustical Society of America, 1977, 62, 1051-1054.

Polzner, H., \& Lane, H. L. Discrimination of location in American Sign Language. In P. Siple (Ed.), Understanding language through sign language research. New York: Academic Press, 1978.

Pols, L., Tramp, H., \& Plomp, R. Frequency analysis of Dutch vowels from 50 male speakers. Journal of the Acoustical Society of America, 1973, 53, 1093-1101.

REPP, B. Dichotic competition of speech sounds: The role of acoustic stimulus structure. Journal of Experimental Psychology: Human Perception and Performance, 1977, 3, 37-50.

Scharf, B., \& LefTon. Backward and forward masking as a function of stimulus and task parameters. Journal of Experimental Psychology, 1970, 84, 331-338.

Selfridge, O. G. Pandemonium: A paradigm for learning. In D. Blake \& A. Utteley (Eds.), Symposium on the mechanization of thought processes. London: H. M. Stationery Office, 1959.

ShePARD, R. N. Analysis of proximities: Multidimensional scaling with an unknown distance function. Psychometrika, 1962, 27, 125-140, 219-246.

She PARD, R. N. Psychological representation of speech sounds. In E. E. Davis \& P. B. Denes (Eds.), Human communication: $A$ unified view. New York: McGraw-Hill, 1972.

Sperling, G. The information available in brief visual presentations. Psychological Monographs, 1960, 74, 1-29.

Sperling, G., \& Speelman, R. G. Acoustic similarity and auditory short-term memory: Experiments and a model. In D. A. Norman (Ed.), Models of human memory. New York: Academic Press, 1970.

Stokoe, W. C., Casterline, D. C., \& Croneberg, C. G. A dictionary of American Sign Language. Washington, D.C: Gallaudet College Press, 1965.

Wickelgren, W. Distinctive features and errors in short-term memory for English vowels. Journal of the Acoustical Society of America, 1965, 38, 583-588.

WickelgreN, W. Distinctive features and errors in short-term memory for English consonants. Journal of the Acoustical Society of America, 1966, 39, 388-398.

Williams, L. Speech perception and production as a function of exposure to a second language. Unpublished doctoral dissertation, Harvard University, 1974.

WoodwARD, J. Implicational variation in American Sign Language. Sign Language Studies, 1974, 5, 20-30.

\section{NOTES}

1. Here the term "distinctive feature" means (and is used interchangeably with) visual cue or visual feature. This is a physical characteristic of the stimulus that is sufficient for its recognition 
as a given segment (as a given handshape, here). The term "distinctive feature" can also mean phonological feature, which is not the intended meaning here. A phonological feature is an abstract dimension in terms of which language users are thought to organize segments once they have been recognized (Parker, 1977, p. 1052). Parker has pointed out, in speech, that many acoustic features signify or "map onto" a single phonological feature. The rules for combining segments in a language are assumed to operate on the small set of phonological features rather than on the myriad of acoustic features. For example, in English there are several different acoustic features that signify the single phonological feature [+voiced]. However, we assume that rather than there being several different phonological rules for plural formation (one for each acoustic feature), just a single rule operates on the phonological feature: if a noun ends in a [+voiced] sound, it takes a [+voiced] plural marker. In sign language, too, it is assumed that the mapping of visual features onto phonological features is many to one and that the phonological rules operate on the phonological distinctive features.

2 . In these matrices, not all columns total 405 . A rechecking of the test tape before final data analysis revealed three mistakes in its construction. The signer performing the test tape from a protocol had misarticulated a trial requiring an $\mathbf{X}$ hand as an $\mathbf{H}$ hand, $a$ bO hand as an $F$, and $a V$ hand as a 3 . Also, one trial for each of the following handshapes was damaged: $\mathbf{C}, \mathbf{R}$, and $\mathrm{L}$. The result is that, pooled over three subjects, five sessions each, there were a maximum of 390 observations for X, bO, V, C, $R$, and $L$ and a maximum of 420 observations for $H, F$, and 3 . When columns do not total 390,420 , or 405 , data are missing due to a subject's not responding after a trial.

3. It is true that use of this similarity score to normalize confusion frequencies and collapse matrices destroys information about asymmetries of misidentification. However, the uncollapsed matrices were not grossly asymmetrical for either signers or nonsigners after the matrices were corrected for response bias. This correction involved first calculating a weight for each row by finding the largest row (response) total and dividing all other row totals into it. Each frequency in a row is then multiplied by its appropriate weight before similarity scores are calculated to collapse the matrix. The correlations between corresponding points of top and bottom halves of uncollapsed pooled matrices were $r=.71$ and $r=.70$ for signers and nonsigners, respectively. (Before the bias correction, these correlations were $r=.57$ and $r=.59$, respectively.) Furthermore, normalizing the confusion frequencies was necessary in order to use hierarchical clustering as means of analyzing the data. The procedure assumes a symmetrical input matrix. Shepard (1972) argues for the use of this similarity score as an appropriate representation of the psychological similarity of speech sounds. He notes, that although there are other theoretical arguments for different definitions of similarity, the formula used here has typically led to the same results and is somewhat less affected by statistical fluctuations in its four components.

4. Consistent patterns in signers' clusterings also necessitated the redefinition of two other features. [+Full] was changed from "four fingers extended" to "four digits extended" (the set of digits includes the thumb, while the set of fingers does not). [+Index] was renamed [+bent] and the definition was changed from "all fingers closed but index" to "just one finger bent at the knuckle or first joint." Also note that [+dual], [+spread], and the renamed $[+$ index] are higher order, more salient features in LBB2 than in LBB1. [+Ulnar], [+full], [+concave], and [+radial] are lower order features. There is no change in $\lfloor+$ compact], [+broad], [+touch], or [+cross].

5. The term "nonredundant" here is used to refer to a feature that does not reiterate class membership. For example, in Figure 2, the four features listed after [compact] are all redundant for handshapes in the [+compact] group, since [+compact] hands are all [-broad], [-dual], [-spread], and [-full]. The first nonredundant feature is [bent], since not all [+compact] hands are plus or minus [bent].
6. Pairs of handshapes could also be assigned a predicted similarity score from the multiple regression equation derived in Lane et al. (1976, pp. 281-284) using coding strategy 4 . In this predicted score, the similarity of pair members depends only on features shared or unshared nonredundantly. Predicted scores for the similarity of each of the three comparison hands within a given standard ranked exactly as the node levels dominating those three pairs. There were two cases in which comparisons did not share more features with the standard as node level increased (X with respect to $O$, and $O$ with respect to $C$ ); predicted similarity did increase, however, because the pairs shared more heavily weighted features according to the multiple regression equation.

7. Note that the members of sign pairs were never identical (or different) on all three parameters: location, orientation, and movement. For each of the 18 handshape pairs within each trial type (12 trials), half the time (6 trials), sign pairs displayed the same primes on two of the three parameters; the other half of the time, sign pairs displayed different primes on two of the three parameters.

8. Before conduction a rank-order correlation of $\mathrm{d}^{\prime}$ with node level, $d^{\prime}$ values were ranked within a handshape standard according to whether they conformed to the rank-order prediction of the model. For $d^{\prime}$ values at the same node level (within or across standards), the binary model makes no claims about the relative order of values and should not be penalized if an ordering exists. Within every handshape standard, then, the lowest $d^{\prime}$ was assigned a rank of 3 , the highest value, a rank of 1 , and the intermediate value (or values, for 0 and 5 standards), a rank of 2. A rank-order correlation was then computed between this set of numbers and the corresponding node levels of the model as predictors. Note that, within a standard, some kinds of $\mathrm{d}^{\prime}$ deviation from node level prediction are more serious than others. A $d^{\prime}$ that is predicted to be the highest but, in fact, turns out to be lowest with respect to a standard will lower RHO considerably more than with an intermediate-high or intermediate-low exchange. Also note that there are 22 handshapes (rather than 18) listed in Table 1. Due to constraints on choosing comparisons of high, intermediate, and low confusability for each of the six standards, the comparisons underlined in Table 1 also appear as standards at the heads of other columns in the table. Since all 22 comparisons were used to evaluate the fit of a model to the discrimination indices, $d$ 's for $O C, O A, 5 B$, and $O B$ entered into the evaluation twice, as they should. For the d' values of these pairs, the model makes rank-order predictions relative to different standard-comparison combinations, depending on which member of the pair is considered to be the standard.

9. Nonmetric multidimensional scaling of signers' and nonsigners' pooled similarity scores was carried out in one to four dimensions. This method of representing similarity structure in confusion data provides a spatial representation of handshape stimuli such that the distances between stimulus pairs vary inversely with the similarity scores of the pairs. The spatial solution is derived from ordinal relations among similarity scores so that uniform expansion, contraction, and rotation of the solution are permissible transformations of it. The scaling program (Version 5M, Kruskal \& Carmone, Note 9) begins a run for one, two, or $\mathrm{N}$ dimensions by setting up the $K$ elements (here $K=20$, the number of handshape primes studied) in a randomly determined starting configuration. The program iteratively adjusts the interpoint distances among the $\mathrm{K}$ elements in successive configurations until stress is at a minimum. Stress is a measure of the goodness of fit of the spatial representation to the similarity scores. The smaller the stress, the more similarity scores vary monotonically with their corresponding interpoint distances in the final configuration of the $K$ elements in $N$ dimensions. A stress value of zero indicates a perfect monotonic relationship between similarity scores and interpoint distances. Stress values for one, two, three, and four dimensions for signers and nonsigners, respectively, were: $.374, .170, .100, .075$, and $.425, .178, .137$, and .078 . A twodimensional scaling solution was chosen to represent both sets of data, since stress decreased relatively little with solutions of three- or 
four-dimensionality and since the dimensions of these solutions were not as clearly interpretable as those of the two-dimensional solution.

10. If we employ redundant binary features, the range and relative discriminability of handshape pairs is the same whether we speak of number of features shared by a pair or the number of features different. Similarly employing continuously variable features, the range and relative discriminability of handshape pairs is the same whether we speak of interpoint proximity of members of a pair or interpoint distance. Employing nonredundant binary features to index a pair's discriminability has no direct analogy in the continuous model. There is a difference in the range and, therefore, the relative discriminability of handshape pairs, depending on whether we speak of number of features shared or number of features different. In LBB2, members of a pair of handshapes can share from zero to four nonredundant features but can differ on only one or two features. While redundant feature assignments are not important to handshape identification, as Lane et al. indicate, if features are processed in binary fashion, redundant features do seem germane to handshape discrimination. The number of shared nonredundant features on an interval scale (in contrast with shared nonredundant features on an ordinal scale employed previously) is not a good predictor of d'. For the 18 pairs of handshapes used in the discrimination study: $r=-.54$ and -.64 for signers and nonsigners, respectively. The number of different nonredundant features is an even worse predictor of $d^{\prime}: r=.13$ for both signers and nonsigners. In contrast, when we include redundant feature specification in calculation of discrimination indices for each of the $\mathbf{1 8}$ handshape pairs (either total number of features same or total number different), we predict $d^{\prime}$ much better: $r=.77$ and .74 for signers and nonsigners, respectively. Following a parsimonious processing strategy, the observer may refrain from using redundant information in identifying handshapes, but makes use of any information available in discriminating them from one another. On this account, discrimination accuracy varies directly with the probability of having extracted a feature difference after the second of a pair of nonsense signs has terminated. If the pair differs on few features, the observer is likely to have extracted only shared features, an incorrect "same" response is made, and discrimination accuracy tends to be low for that pair. If the pair differs on many features, the probability of having extracted an unshared feature is increased and discrimination accuracy tends to be high.

11. Orthogonal $X$ and $Y$ axes were arbitrarily placed with respect to the spatial configuration of handshapes in Figure 3. $X$ and $Y$ coordinates for a point corresponding to each handshape were read from these axes. The coordinates employed for each handshape are as follows:

\begin{tabular}{crrrrr}
$\begin{array}{c}\text { Hand- } \\
\text { shape }\end{array}$ & \multicolumn{1}{c}{ X } & Y & $\begin{array}{c}\text { Hand- } \\
\text { shape }\end{array}$ & \multicolumn{1}{c}{ X } & \multicolumn{1}{c}{ Y } \\
O & -1.30 & 3.20 & W & 1.80 & -2.85 \\
C & .50 & 3.59 & Y & .00 & .00 \\
bO & -2.65 & .16 & I & .00 & 1.00 \\
X & -2.40 & -.35 & V & -.25 & -3.08 \\
E & -1.30 & 1.89 & R & -.90 & -3.00 \\
A & -2.68 & .69 & H & -.60 & -3.10
\end{tabular}

$\begin{array}{llrlrr}5 & 3.65 & -.38 & \text { L } & -1.00 & -.89 \\ \text { B } & 3.10 & 2.13 & \text { G } & -1.80 & -.80 \\ 8 & 2.50 & -2.39 & 3 & .00 & -3.19 \\ \text { F } & 2.10 & -2.68 & \text { K } & -1.80 & -2.47\end{array}$

Each interpoint distance in Figure 3 is a vector whose length and $X, Y$ coordinates are calculated from the $X, Y$ coordinates of the two endpoints that define the distance. Using the $X, Y$ coordinates for each of two vectors that share an endpoint, the following formula can be employed to find the cosine of the angle formed by the vectors directly without first knowing the angle measurement:

Let $\overrightarrow{\mathrm{OX}}$ correspond to vector $\mathrm{V}$, whose coordinates are found by subtracting the corresponding coordinates of its endpoints:

$$
\mathrm{V}=\left(\mathrm{X}_{\mathrm{X}}-\mathrm{X}_{\mathrm{O}}, \mathrm{Y}_{\mathrm{X}}-\mathrm{Y}_{\mathrm{O}}\right)=\left(\mathrm{X}_{\mathrm{V}}, \mathrm{Y}_{\mathrm{V}}\right) \text {. }
$$

Let $\overrightarrow{O A}$ correspond to vector $W$, whose coordinates are found similarly:

$$
W=\left(X_{A}-X_{O}, Y_{A}-Y_{O}\right)=\left(X_{W}, Y_{W}\right)
$$

Then

$$
\cos (\mathrm{LXOA})=\frac{\left(\mathrm{X}_{\mathrm{V}} \cdot \mathrm{X}_{\mathrm{W}}\right)+\left(\mathrm{Y}_{\mathrm{V}} \cdot \mathrm{Y}_{\mathrm{W}}\right)}{\sqrt{\left(\mathrm{X}_{\mathrm{V}}\right)^{2}+\left(\mathrm{Y}_{\mathrm{V}}\right)^{2}}+\sqrt{\left(\mathrm{X}_{\mathrm{W}}\right)^{2}+\left(\mathrm{Y}_{\mathrm{W}}\right)^{2}}} .
$$

12. Six of the $\mathbf{3 8}$ predictions made by the continuous model cannot be made by the binary model additively. For example, no prediction of a $d^{\prime}$ value for FB is possible using obtained $d^{\prime}$ values of $F 5$ and $5 B$. Unlike 32 of the 38 predictions, members of

\begin{tabular}{|c|c|c|c|}
\hline $\begin{array}{l}\text { pair } \\
\text { features different }\end{array}$ & $\begin{array}{l}\text { F5 } \\
\text { full } \\
\text { touch } \\
\text { radial }\end{array}$ & \pm & $\begin{array}{l}5 B \\
\text { spread } \\
\text { radial }\end{array}$ \\
\hline
\end{tabular}
the pair FB are not differentiated by the sum or difference of the features differentiating F5 and 5B. For example,

13. Multiple regression analyses were carried out using the similarity scores of signers, nonsigners, and Lane et al.'s signers as dependent variables and the features of $L B B 2$ as predictor variables. Of the four coding strategies advanced by Lane et al. (1976, pp. 281-282), coding strategy 4 resulted in the highest multiple Rs. These were $.74, .75$, and .65 for signers, nonsigners, and Lane et al.'s signers, respectively. When the beta weights from the deaf multiple regression analysis are attached to the features different, LBB2 predicts even more strongly that the $\mathrm{BO}$ and $\mathrm{BE}$ discrimination indices should exceed $\mathbf{8 B}$. So it is not the case that 8 and $B$ differ by three heavily weighted features while $\mathrm{BO}$ and $\mathrm{BE}$, respectively, differ by six and five low-level features. If this were so, it could make the binary model consistent with the data.

(Received for publication July 17, 1979; revision accepted November 17,1980 .) 